\title{
Diabetic neuropathy: are we still barking up the wrong tree and is change finally in sight?
}

\author{
David V. Coppini ${ }^{1}$
}

Received: 10 June 2020 / Accepted: 12 June 2020 / Published online: 17 July 2020

(C) Springer-Verlag GmbH Germany, part of Springer Nature 2020

Keywords Artificial intelligence $\cdot$ Diabetic neuropathy $\cdot$ Future

To the Editor: I read with interest the review article by Callaghan et al on diabetic neuropathy that was recently published in Diabetologia [1]. One seemingly relentless problem with this elusive diabetes complication is that recommended diagnostic methods [2] are much more applicable to research rather than to everyday clinical practice. As a result, diagnostic guidelines for diabetic neuropathy within diabetes clinic settings remain widely variable. Its heterogeneous presentation and insidious natural history render diabetic neuropathy research equally problematic. Clinical trials investigating a therapeutic role for protein kinase $\mathrm{C} \beta(\mathrm{PKC} \beta)$ inhibition, nerve growth factor (NGF) and aldose reductase inhibition [3] in established neuropathy using robust diagnostic criteria have been largely disappointing, and effective licensed treatments are still unavailable. The role of metabolic factors, such as lipids and sphingolipids, in the aetiology of neuropathy is discussed in some detail in the review by Callaghan and colleagues [1]. Although of novel scientific interest, further research in this area is realistically unlikely to influence clinical practice. Despite observed associations between dyslipidaemia and neuropathy, the limited outcome studies on lipid-lowering therapies in diabetic neuropathy are both unconvincing and conflicting [4]. The interventional research studies targeting obesity that are proposed by Callaghan et al [1] may show an interesting positive effect on neural function but, in the real world, weight loss and lifestyle modification remain a key strategic measure in diabetes irrespective of their effect on neuropathy. As prediabetes (impaired fasting glucose and impaired glucose tolerance), which is often related to obesity, is related to early complications, including neuropathy [5], investment in diabetes

David V. Coppini

Dcoppini@aol.com

1 Mater Dei Hospital, Dun Karm Street, L-Imsida MSD2090, Malta prevention programmes would seem a much safer direction. After all, the past has taught us with some conviction that prevention of neuropathy (as shown by the DCCT and, to a lesser extent, by the UK Prospective Diabetes Study [UKPDS]), is a much more reliable option than treatment.

Unsurprisingly, patients' understanding of neuropathy remains nebulous when compared with that of the other microvascular complications of diabetes, particularly in those who are asymptomatic. And in those with symptoms, particularly those troubled with severe neuropathic pain, therapeutic options also remain disappointing, as is well highlighted by Callaghan and colleagues [1]. However, the importance of a specialist team in managing painful neuropathy needs to be emphasised, as, in our experience, patient education and psychological support are invaluable in complementing otherwise suboptimal pharmacotherapies [6]. Whilst we should certainly not underestimate the wealth of scientific research in the last two decades, clinically relevant outcomes have been lacking. With the increasing worldwide prevalence of diabetes, and of diabetic neuropathy, there is a need for quicker, more intuitive diagnostic devices. Smart artificial-intelligence systems are making good headway in diabetes research and provide a new platform for a long-awaited 'one-language' approach to diagnosing and managing diabetic neuropathy [7]. Our own ongoing research in this area, utilising simple standard clinical variables, is certainly very encouraging [8]. As recent events related to coronavirus disease-2019 (COVID-19) continue to unfold, changes in clinical practice are inevitable [9] and practical, low- or non-contact techniques provided by medical artificial-intelligence systems will almost certainly become the way of the 'new-normal' future.

Authors' relationships and activities The author declares that there are no relationships or activities that might bias, or be perceived to bias, their work.

Contribution statement The author was the sole contributor to this paper. 


\section{References}

1. Callaghan BC, Gallagher G, Fridman V, Feldman EL (2020) Diabetic neuropathy: what does the future hold? Diabetologia 63: 891-897. https://doi.org/10.1007/s00125-020-05085-9

2. Pop-Busui R, Boulton AJM, Feldman E et al (2017) A position statement by the American Diabetes Association. Diabetes Care 40(1):136-154

3. Tentolouris N, Alexiadou K, Makrilakis K, Liatis S, Jude E, Boulton AJ (2014) Standard and emerging treatment options for diabetic neuropathy. Curr Pharm Des 20(22):3689-3704. https://doi.org/10. 2174/13816128113196660682

4. Jende JME, Groener JB, Rother C et al (2019) Association of serum cholesterol levels with peripheral nerve damage in patients with type 2 diabetes. JAMA Netw Open 2(5):e194798. https://doi.org/10. 1001/jamanetworkopen.2019.4798

5. Ziegler D, Rathmann W, Dickhaus T, Meisinger C, Mielck A (2008) Prevalence of polyneuropathy in pre-diabetes and diabetes is associated with abdominal obesity and macroangiopathy: the MONICA/ KORA Augsburg Surveys S2 and S3. Diabetes Care 31(3):464-469. https://doi.org/10.2337/dc07-1796
6. Coppini DV (2016) Enigma of painful diabetic neuropathy: can we use the basic science, research outcomes and real-world data to help improve patient care and outcomes? Diabet Med 33(11):1477-1482. https://doi.org/10.1111/dme.13089

7. Williams BM, Borroni D, Liu R et al (2020) An artificial intelligence based deep learning algorithm for the diagnosis of diabetic neuropathy using corneal confocal microscopy: a development and validation study. Diabetologia 63:419-430. https://doi.org/10.1007/ s00125-019-05023-4

8. Dave J, Dubey VN, Coppini DV, Beavis J (2019) Predicting diabetic neuropathy risk level using artificial neural network based on clinical characteristics of subjects with diabetes. Diabet Med 36(S1):144 (Abstract)

9. Thornton J (2020) Covid19: how coronavirus will change the face of general practice forever. BMJ 368:m1279. https://doi.org/10.1136/ bmj.m1279

Publisher's note Springer Nature remains neutral with regard to jurisdictional claims in published maps and institutional affiliations. 